\title{
Electron and Hole States in Closed Spherical Quantum Dot with Linearly Graded Composition
}

\author{
R. Kostić And D. Stojanović* \\ Institute of Physics, Pregrevica 118, 11080 Belgrade, Serbia
}

\begin{abstract}
The theoretical investigation of the electron and hole spectra in a quantum dot with a linearly graded composition within the effective mass approximation is presented. The particular example is $\beta$-HgS surrounded by CdS. $\beta-\mathrm{HgS}$ core of radius $r_{\mathrm{C}}$ is surrounded by concentric spherical layers each of $\mathrm{Hg}_{1-x} \mathrm{Cd}_{x} \mathrm{~S}$ composition ( $x$ is function of $r$ ) and finally, form radius $r_{\mathrm{S}}$ by CdS. The existence of these intermediate layers, as model of graded composition, influences rapidly electron and hole spectra.
\end{abstract}

PACS numbers: 73.22.Dj, 73.21.La

\section{Introduction}

Physical properties of quantum dots and related compounds have become under intensive investigation for potentials to use in optoelectronics, high density memory, quantum dot lasers, biosensing and biolabeling.

Continuous advances have led to the development of various heterostructures. The choice of materials and their space arrangement is determined by the desired properties.

At the surface between two materials there is a drastic change in composition and in all parameters. Technologically it is not always possible to get such a step-like change in composition, and a graded composition space is often formed between two materials. In some cases this grade composition is designed to get the desired optical properties without size changing [1-5].

Spherical nano-heterosystems $\mathrm{HgS} / \mathrm{CdS}$ have been theoretically [6-9] and experimentally $[6,10,11,12]$ intensively studied. In this paper we present calculation results for spherical quantum dot that consists of: a spherical core of $\beta$ - HgS surrounded by $N$ layers of $\mathrm{Hg}_{1-x} \mathrm{Cd}_{x} \mathrm{~S}$ compositions ( $x$ is proportional to the layer distance from the center) and finally by CdS. It is well known that CdS and $\mathrm{HgS}$ readily form solid solutions on their entire concentration range.

Our calculations were performed in the well-known and widely used effective mass approximation (EMA) [6-8]. The parameters for the calculation in EMA are effective masses of materials in the structure and conduction and valence offsets between materials. We assumed that effective masses and conduction and valence offsets change proportionally to $x$, from the $\mathrm{HgS}$ values to the $\mathrm{CdS}$ values.

\footnotetext{
* corresponding author; e-mail: dusanka@phy.bg.ac.yu
}

\section{Model and results}

A spherical heterosystem consisting of a $\beta$ - $\mathrm{HgS}$ core with the radius $r_{\mathrm{C}}$ covered by $N \mathrm{Hg}_{1-x} \mathrm{Cd}_{x} \mathrm{~S}$ layers embedded in CdS with the radius $r_{\mathrm{S}}$, is investigated. In this particular case $N=4$. The $i$-th layer, $i=1, \ldots, N$, consists of $\operatorname{Hg}_{1-x} \mathrm{Cd}_{x} \mathrm{~S}, x=i /(N+1)$ and is situated between radius $r_{i-1}$ and radius $r_{i}$ :

$$
r_{i}= \begin{cases}r_{0}=r_{\mathrm{C}}, & i=0, \\ \frac{r_{\mathrm{S}}-r_{\mathrm{C}}}{N} i+r_{\mathrm{C}}, & i=1, \ldots, N .\end{cases}
$$

Electrons and holes in such a system are characterized by their effective masses and potentials. Effective masses are

$$
\begin{aligned}
& m_{\mathrm{e}, \mathrm{h}}^{*}(r)= \\
& \begin{cases}m_{\mathrm{e}, \mathrm{h}_{\mathrm{o}}}^{*},-m_{\mathrm{e}, \mathrm{h}_{0}}^{*} i+m_{\mathrm{e}, \mathrm{h}_{0}}^{*}, & r_{i-1}<r \leq r_{i}, \\
\frac{m_{\mathrm{e}, \mathrm{h}_{N+1}}}{N+1} & i=1, \ldots, N, \\
m_{\mathrm{e}, \mathrm{h}_{N+1}}^{*}, & r>r_{N}=r_{\mathrm{S}} .\end{cases}
\end{aligned}
$$

In this case $m_{\mathrm{e}, \mathrm{h}_{0}}^{*}$ are effective masses of $\mathrm{HgS}\left(m_{\mathrm{e}_{0}}^{*}=\right.$ $\left.m_{\mathrm{e}_{\mathrm{HgS}}}^{*}=0.036 m_{\mathrm{e}}, m_{\mathrm{h}_{0}}^{*}=m_{\mathrm{h}_{\mathrm{HgS}}}^{*}=-0.044 m_{\mathrm{e}}\right)$ and $m_{\mathrm{e}, \mathrm{h}}^{*}{ }_{N+1}$ are effective masses of CdS $\left(m_{\mathrm{e}_{N+1}}^{*}=m_{\mathrm{e}_{\mathrm{CdS}}}^{*}=\right.$ $0.2 m_{\mathrm{e}}, m_{\mathrm{h}_{N+1}}^{*}=m_{\mathrm{h}_{\mathrm{CdS}}}^{*}=-0.7 m_{\mathrm{e}}$ ) (see Table). Potentials are

$$
\begin{aligned}
& V_{\mathrm{e}, \mathrm{h}}(r)= \\
& \begin{cases}V_{\mathrm{e}, \mathrm{h}_{0}}, & r \leq r_{0}=r_{\mathrm{C}}, \\
\frac{V_{\mathrm{e}, \mathrm{h}_{N+1}}-V_{\mathrm{e}, \mathrm{h}_{0}}}{N+1} i+V_{\mathrm{e}, \mathrm{h}_{0}}, & r_{i-1}<r \leq r_{i}, \\
& i=1, \ldots, N, \\
V_{\mathrm{e}, \mathrm{h}_{N+1}}, & r>r_{N}=r_{\mathrm{S}} .\end{cases}
\end{aligned}
$$

In this case $V_{\mathrm{e}, \mathrm{h}_{0}}$ are electron and hole potentials in $\operatorname{HgS}\left(V_{\mathrm{e}_{0}}=V_{\mathrm{e}_{\mathrm{HgS}}}=-1.35 \mathrm{eV}, V_{\mathrm{h}_{0}}=V_{\mathrm{h}_{\mathrm{HgS}}}=-1.85 \mathrm{eV}\right)$, and $V_{\mathrm{e}, \mathrm{h}_{N+1}}$ are electron and hole potentials in CdS 


\section{TABLE}

Material parameters of the system: $a$ - lattice constant, $E_{\mathrm{g}}$ - energy gap, $V_{\mathrm{e}}, V_{\mathrm{h}}-$ conduction and valence band potential (energetic scale is $V_{\mathrm{e}}=0 \mathrm{eV}$ in $\mathrm{CdS}), m^{*}$ - effective mass, $m_{\mathrm{e}}$ - electron mass.

\begin{tabular}{c|c|c|c|c|c|c}
\hline \hline & $a[\AA]$ & $E_{\mathrm{g}}[\mathrm{eV}]$ & $V_{\mathrm{e}}[\mathrm{eV}]$ & $V_{\mathrm{h}}[\mathrm{eV}]$ & $m_{\mathrm{e}}^{*} / m_{\mathrm{e}}$ & $m_{\mathrm{h}}^{*} / m_{\mathrm{e}}$ \\
\hline$\beta-\mathrm{HgS}$ & 5.851 & 0.5 & -1.35 & -1.85 & 0.036 & -0.044 \\
$\mathrm{CdS}$ & 5.818 & 2.5 & 0 & -2.5 & 0.2 & -0.7
\end{tabular}

$\left(V_{e_{N+1}}=V_{\mathrm{e}_{\mathrm{CdS}}}=0 \mathrm{eV}, V_{\mathrm{h}_{N+1}}=V_{\mathrm{h}_{\mathrm{CdS}}}=-2.5 \mathrm{eV}\right)$, Table.

Considering that electron and hole spectra are mainly formed by size quantization, the stationary Schrödinger equation for a single particle, in this case, may be expressed as

$$
\left(-\frac{\hbar^{2}}{2 m^{*}} \nabla^{2}+V(r)\right) \Psi(r)=E \Psi(r) .
$$

For spherically symmetric potential $V(r)$ the separation of radial and angular coordinates leads to

$$
\Psi_{n l m}(r)=R_{n l}(r) Y_{l m}(\theta, \varphi) .
$$

$R_{n l}(r)$ is the radial wave function, and $Y_{l m}(\theta, \varphi)$ is a spherical harmonic. $n$ is the principal quantum number, and $l$ and $m$ are the angular momentum quantum numbers.

For a spherical potential with stepwise constant values $V_{0}$ (in core), $V_{q}(q=1, \ldots, N)$ and $V_{N+1}$ in surrounding material, as defined in (3), the radial function $R_{n l, q}(r)$ in energy region $\left|V_{q}\right|>|E| \geq\left|V_{q+1}\right|, q=0, \ldots, N$ consist of $N+2$ parts

$$
R_{n l, q}=\left\{\begin{array}{c}
R_{n l, q}^{0}=A_{0}^{l} j_{l}\left(K_{0} r\right), \\
\quad r \leq r_{\mathrm{C}}=r_{0}, \\
R_{n l, q}^{i}=A_{i}^{l} j_{l}\left(K_{i} r\right)+B_{i}^{l} n_{l}\left(K_{i} r\right), \\
r_{i-1} \leq r \leq r_{i}, i=1, \ldots, N, \\
R_{n l, q}^{N+1}=A_{N+1}^{l} h_{l}^{(1)}\left(K_{N+1} r\right) \\
r \geq r_{N}=r_{\mathrm{S}},
\end{array}\right.
$$

$K_{0}=\sqrt{\frac{2 m_{0}^{*}}{\hbar^{2}}\left(\left|V_{0}\right|-|E|\right)}, K_{i}=\sqrt{\frac{2 m_{i}^{*}}{\hbar^{2}}\left(\left|V_{i}\right|-|E|\right)}, i=$ $1, \ldots N, K_{N+1}=\sqrt{\frac{2 m_{N+1}^{*}}{\hbar^{2}}(-|E|)}, j_{l}, n_{l}, h_{l}^{(1)}$ are the Bessel, Neumann and Hankel spherical functions. Solutions already satisfy conditions to be regular when $r=0$ and to vanish sufficiently rapidly when $r \rightarrow \infty$. The solution must satisfy boundary conditions

$$
\begin{aligned}
& \left.R_{n l, q}^{i}(r)\right|_{r=r_{i}}=\left.R_{n l, q}^{i+1}(r)\right|_{r=r_{i}}, \\
& \left.\frac{1}{m_{i}^{*}} \frac{\mathrm{d} R_{n l, q}^{i}(r)}{\mathrm{d} r}\right|_{r=r_{i}}=\left.\frac{1}{m_{i+1}^{*}} \frac{\mathrm{d} R_{n l, q}^{i+1}(r)}{\mathrm{d} r}\right|_{r=r_{i}} \\
& \quad i=0, \ldots, N .
\end{aligned}
$$

Equations (7) for each $q, q=0, \ldots, N$, lead to a system of $2 N+2$ linear equations for the $2 N+2$ unknown coefficients. It has nontrivial solutions only if its determinant

$$
D_{l, q}=D_{l, q}\left(E_{n l}\right)=0, \quad\left|V_{q}\right|>|E| \geq\left|V_{q+1}\right|,
$$

$$
q=0, \ldots, N .
$$

Once the eigenvalues $E_{n l}$ are determined from (8), the linear equations can be solved yielding the coefficients to be a function of one of them. The last undetermined coefficient is determined by the normalization condition for $R_{n l, q}(r), \int_{0}^{\infty} R_{n l, q}^{2}(r) r^{2} \mathrm{~d} r=1$. As $q$ goes from 0 to $N$ and all solutions are determined, we can unify them to get the complete picture of eigensolutions $E_{n l}$ and corresponding wave functions $R_{n l}$. These calculations were performed independently for electrons and holes (procedure is similar), giving the confinement energies $E_{n l}^{\mathrm{e}}$ and $E_{n l}^{\mathrm{h}}$, and wave functions $R_{n l}^{\mathrm{e}}$ and $R_{n l}^{\mathrm{h}}$.

The calculations of the electron and hole spectra in the heterosystem under study were performed according to the described model with the common material parameters of the system, given in Table $[6,7]$. We investigated the influence of width of grade region to transition energies for a QD of fixed dimension. We illustrate this influence through example: $r_{\mathrm{S}}=10 a_{0}=5.851 \mathrm{~nm}$ $\left(a_{0}=a_{\mathrm{HgS}}\right)$ and present electron and hole spectra in Fig. $1, l=0,1,2$.

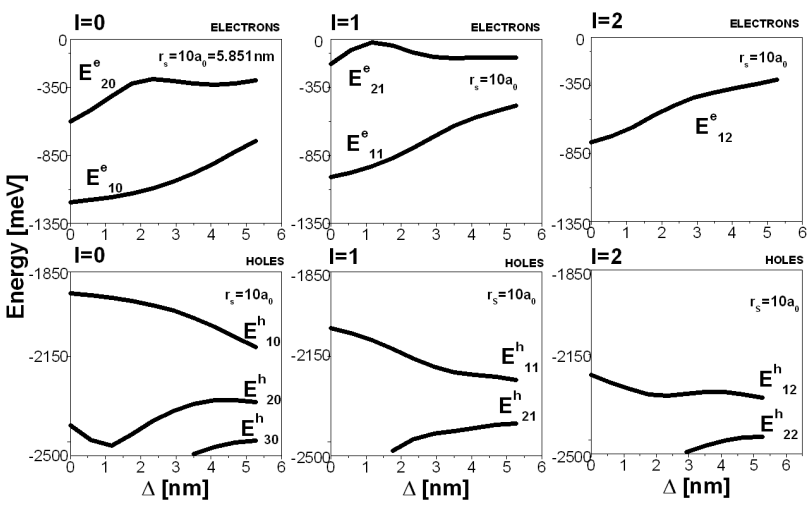

Fig. 1. Electron and hole spectra $l=0,1,2$ for $r_{\mathrm{S}}=$ $10 a_{0}=5.851 \mathrm{~nm}$ as function of $\Delta$ (the width of the sector of gradient composition).

$\Delta=r_{\mathrm{S}}-r_{0}$ is the width of the spherical shell of grade $\mathrm{Hg}_{1-x} \mathrm{Cd}_{x} \mathrm{~S}$ composition. $r_{0}=r_{\mathrm{S}}-\Delta$ is the radius of the $\mathrm{HgS}$ core. $\Delta=0$ is a case of the $\mathrm{HgS}$ sphere with the radius $r_{0}=r_{\mathrm{S}}$, i.e. without gradient region, surrounded by $\mathrm{CdS}$. This is a well known structure and a basic example of a closed QD.

Each $\Delta$ value in Fig. 1 corresponds to nano-heterosystem of characteristic gradual composition in region between the $\mathrm{HgS}$ core and the surrounding $\mathrm{CdS}$ medium. Bigger $\Delta$ corresponds to smaller gradient of composition within $\Delta$.

The energies of the first solutions for electrons $E_{10}^{\mathrm{e}}$, $E_{11}^{\mathrm{e}}, E_{12}^{\mathrm{e}}$ increase and the first solutions for holes $E_{10}^{\mathrm{h}}$, $E_{11}^{\mathrm{h}}, E_{12}^{\mathrm{h}}$ decrease with the increase in $\Delta$. Energy of basic transition, between the first hole and the first electron state, is $E_{10}(\Delta)=E_{10}^{\mathrm{e}}(\Delta)-E_{10}^{\mathrm{h}}(\Delta) . E_{10}(\Delta)$ energy increases from $E_{10}(\Delta=0)=731 \mathrm{meV}$ to $E_{10}\left(\Delta \rightarrow r_{\mathrm{S}}\right) \rightarrow$ $1480 \mathrm{meV}$. Therefore, the energy of the basic transition is increased by $\approx 760 \mathrm{meV}$. 
From corresponding wave functions one can see that the electron and the hole are situated at almost the same position in QD. When $\Delta$ increases both the electron and the hole move towards the center of the $\mathrm{QD}$, to be in the core as much as possible.

For comparison, the results of our energy calculations for $r_{\mathrm{S}}=5 a_{0}=2.9255 \mathrm{~nm}$ of basic transitions are $E_{10}(\Delta=0)=1053 \mathrm{meV}$ (for smaller $r_{0}=r_{\mathrm{S}}$ transition energy is higher) and $E_{10}\left(\Delta \rightarrow r_{s}\right) \rightarrow 1875 \mathrm{meV}$. The energy of the basic transition is increased by $\approx 820 \mathrm{meV}$. Like in the first example, when $\Delta$ increases both the electron and the hole move towards the center of the QD, but, as there is less space than in the first example, they are forced to situate in the $\Delta$ region. In this case, in the gradual composition region the potential is less deep than in the core and particles tend to move into the core. In smaller QD, where space is more limited, change of transition energies is more drastic for the same $\Delta$ values.

\section{Discussion and conclusions}

To illustrate the influence of inhomogeneity to characteristic energies we combined $\mathrm{HgS}$ and $\mathrm{CdS}$ and formed the spherical heterosystem $\mathrm{HgS} / \mathrm{Hg}_{1-x} \mathrm{Cd}_{x} \mathrm{~S} / \mathrm{CdS}$. Various heterosystems formed by these materials were investigated in detail, but never in this composition.

In this particular case the existence of gradual composition region for a case of fixed QD dimension, $r_{\mathrm{S}}$, influences drastically basic $E_{10}$ transition energy.

\section{Acknowledgments}

This work was supported by Serbian Ministry of Science (Projects No. 141028 and No. 141047).

\section{References}

[1] C. Dion, P. Desjardins, N. Shtinkov, M.D. Robertson, F. Schiettekatte, P.J. Poole, S. Raymond, Phys. Rev. B 77, 075338 (2008).

[2] P.M. Koenraad, D.M. Bruls, J.H. Davies, S.P.A. Grill, Fei Long, M. Hopkinson, M. Skolnick, J.H. Wolter, Physica E 17, 526 (2003).

[3] C.L.N. Oliveira, J.A.K. Freire, V.N. Freire, G.A. Farias, Appl. Surf. Sci. 237, 266 (2004).

[4] H.J. Chu, J. Wang, J. Appl. Phys. 98, 034315 (2005).

[5] R.E. Bailey, J.B. Strausburg, S. Nie, J. Nanosci. Nanotechnol. 4, 569 (2003).

[6] D. Schooss, A. Mews, A. Eychmuller, H. Weller, Phys. Rev. B 49, 17072 (1994).

[7] N.V. Tkach, V.A. Golovatskii, O.M. Voitsekhivskaya, M. Ya. Mikhal'ova, R.B. Fartustinskii, Phys. Solid State 43, 1315 (2001).

[8] N.V. Tkach, Yu. A. Sety, Semiconductors 40, 1083 (2006).

[9] E.P. Pokatilov, V.A. Fonoberov, V.M. Fomin, J.T. Devreese, Phys. Rev. B 64, 245329 (2001).

[10] A.V. Yeh, G. Cerullo, U. Banin, A. Mews, A.P. Alivisatos, C.V. Shank, Phys. Rev. B 59, 4973 (1999).

[11] F. Koberling, A. Mews, T. Basche, Phys. Rev. B 60, 1921 (1999).

[12] M. Braun, S. Link, C. Burda, M. El-Sayed, Phys. Rev. B 66, 205312 (2002). 